\title{
Physician Mothers and Breastfeeding: A Cross-Sectional Survey
}

\author{
Maryam Sattari,, David M. Levine, ${ }^{2}$ Lazarus K. Mramba, ${ }^{3}$ Monica Pina, ${ }^{4}$ Reet Raukas, ${ }^{5}$ \\ Elien Rouw, ${ }^{6}$ and Janet R. Serwint ${ }^{7}$
}

\begin{abstract}
Objectives: To explore infant-feeding intentions and behavior of physician mothers as well as their breastfeeding enablers and obstacles.

Study Design: A cross-sectional online survey was conducted among female physicians with at least one biological child recruited through the Academy of Breastfeeding Medicine. The main outcomes were duration of exclusive breastfeeding (EBF) and duration of any breastfeeding (BFD). We determined predictors of EBF and BFD.

Results: The 570 participants reported intention to breastfeed at least 12 months in $78.1 \%$ of cases. Breastfeeding rates were $97.8 \%, 85.5 \%$, and $55.4 \%$ at birth, 6, and 12 months. EBF rates were 88.5\%, 76.3\%, and $40.9 \%$ at birth, 3, and 6 months. Younger participant age, breastfeeding discontinuation not due to work-related demands, and heightened maternal satisfaction with BFD were associated with longer EBF and BFD. EBF at birth, less maternal stress, availability of time to express milk, and collegial support were associated with longer EBF. Longer maternal BFD goal, longer maternity leave, existence of laws or regulations to support breastfeeding among working mothers, later child order, and lower level of maternal depression were associated with longer BFD.

Conclusions: Maternal infant-feeding intentions and work-related factors both play important roles in physician mothers' infant-feeding behavior. Longer maternity leave, regulations to support breastfeeding among working mothers, and workplace support might significantly improve physician mothers' BFD.
\end{abstract}

Keywords: infant-feeding intention, physician mothers, breastfeeding duration, exclusive breastfeeding

\section{Introduction}

B REASTFEEDING IS THE UNIVERSALLY recommended modality of infant feeding. ${ }^{1-4}$ Current recommendations are exclusive breastfeeding $(\mathrm{EBF})$ for the first 6 months of life, followed by continued breastfeeding throughout at least the first year. ${ }^{1,3} \mathrm{EBF}$ is defined as infant receiving only breast milk and no other liquids or solids except vitamins, minerals, or medications. ${ }^{1}$ Physicians' breastfeeding advice effectively increases patients' breastfeeding initiation and continuation. ${ }^{5}$ The strongest predictor of physicians' clinical breastfeeding advocacy is their personal or spousal breastfeeding behavior. $^{6-8}$ Therefore, studying physician mothers' breastfeeding behavior is important as it impacts their anticipatory guidance to patients and, therefore, influences patients' breastfeeding behavior.

Despite excellent breastfeeding initiation rates, female physicians in the United States seem to also be at risk of premature breastfeeding cessation before achieving their own individual breastfeeding duration (BFD) goal. ${ }^{9-14}$ Enabling physician mothers to achieve their personal BFD goals represents an opportunity to improve not only the health of these physicians and their families, but ultimately the health of their patients and patients' families as well. ${ }^{10}$ Identification

${ }^{1}$ Division of General Internal Medicine, Department of Medicine, University of Florida College of Medicine, Gainesville, Florida, USA.

${ }^{2}$ Department of Medicine, Johns Hopkins University School of Medicine, Baltimore, Maryland, USA.

${ }^{3}$ Department of Biostatistics and Data Science, University of Kansas Medical Center, Kansas City, Kansas, USA.

${ }_{5}^{4}$ Department of Internal Medicine, Clínica Planalto, Lisbon, Portugal.

${ }^{5}$ Department of Neonatology, East-Tallinn Central Hospital, Tallinn, Estonia.

${ }^{6}$ Academy of Breastfeeding Medicine, Bühl, Germany.

${ }^{7}$ Department of Pediatrics, Johns Hopkins University School of Medicine, Baltimore, Maryland, USA. 
Table 1. Characteristics of Study PARTICIPANTS $(N=570)$

\begin{tabular}{lrr}
\hline Variable & $\mathrm{n}$ & \multicolumn{1}{c}{$\%$} \\
\hline Participant age (years) at the time of the survey & \\
$20-29$ & 21 & 3.68 \\
$30-39$ & 439 & 77.02 \\
$40-49$ & 96 & 16.84 \\
$50+$ & 14 & 2.46
\end{tabular}

Maternal country of residence at the time of the study

Canada

Costa Rica

Ireland

Israel

Puerto Rico

Spain

Sweden

The United States

Missing response

Marital status

Married

Not married (single living with/without domestic partner, separated, divorced, widowed)

Missing response

Medical specialty

Anesthesiology

Dermatology

Emergency medicine

ENT

Family medicine

General surgery

Internal medicine

Internal medicine/pediatrics

Neurology

Obstetrics and gynecology

Ophthalmology

Orthopedics

Pediatrics

Physical medicine and rehabilitation

Preventive medicine

Psychiatry

Radiology

Urology

Other

Missing response

\begin{tabular}{rr}
10 & 1.75 \\
1 & 0.18 \\
1 & 0.18 \\
2 & 0.35 \\
1 & 0.18 \\
1 & 0.18 \\
1 & 0.18 \\
549 & 96.32 \\
4 & 0.70 \\
& \\
535 & 93.86 \\
25 & 4.39 \\
& \\
& \\
10 & 1.75 \\
& \\
16 & 2.81 \\
11 & 1.93 \\
35 & 6.14 \\
3 & 0.53 \\
85 & 14.91 \\
25 & 4.39 \\
58 & 10.18 \\
15 & 2.63 \\
8 & 1.40 \\
66 & 11.58 \\
12 & 2.11 \\
5 & 0.88 \\
109 & 19.12 \\
5 & 0.88 \\
1 & 0.18 \\
18 & 3.16 \\
16 & 2.81 \\
3 & 0.53 \\
55 & 9.65 \\
24 & 4.21 \\
& \\
97 & 17.02 \\
469 & 82.28 \\
4 & 0.70 \\
& \\
218 & 38.25 \\
243 & 42.63 \\
90 & 15.79 \\
18 & 3.16 \\
1 & 0.18 \\
\hline & \\
& \\
& \\
& \\
& \\
& \\
& \\
& \\
& \\
& \\
& \\
& \\
& \\
&
\end{tabular}

Career stage at the time of study

In training

In practice (completed training)

Missing response

No. of children

1

2

3

4

Policies and Laws Pertaining to Maternal and Child Health

Do you know if your country has a formal maternity leave policy? (Maternity Leave Policy)

$\begin{array}{lrr}\text { Yes } & 334 & 58.60 \\ \text { No } & 142 & 24.91 \\ \text { Don't know } & 94 & 16.49\end{array}$

(continued)
TABle 1. (CONTINUED)

\begin{tabular}{lrr}
\hline Variable & $\mathrm{n}$ & $\%$ \\
\hline \multicolumn{2}{c}{ Does your country have any laws or regulations to } \\
encourage breastfeeding among working mothers? \\
(BF Work laws) \\
Yes & 245 & 42.98 \\
No & 176 & 30.88 \\
Don't know & 148 & 25.96 \\
Missing & 1 & 0.18
\end{tabular}

Breastfeeding Advocacy

With a typical pregnant patient or new mother, how often do you actually talk with them about breastfeeding? (BF Discussion)

$\begin{array}{lll}\text { Usually/always } & 338 & 59.30 \\ \text { Sometimes } & 114 & 20.00 \\ \text { Never/rarely } & 102 & 17.89\end{array}$

$\begin{array}{lrr}\text { Never/rarely } & 102 & 17.89 \\ \text { Missing } & 16 & 2.81\end{array}$

How strongly do you encourage pregnant patients or new mothers to breastfeed? (BF Encouragement)

Strongly encourage $\quad 208 \quad 36.49$

$\begin{array}{lll}\text { Encourage } & 227 & 39.82\end{array}$

$\begin{array}{lll}\text { Neither encourage nor discourage } & 116 & 20.35\end{array}$

Discourage/strongly discourage $\quad 7 \quad 1.23$

$\begin{array}{lll}\text { Missing } & 12 & 2.11\end{array}$

Do you feel that you actively promote breastfeeding among your women patients? (BF Promotion)

$\begin{array}{lll}\text { Yes } & 420 & 73.68\end{array}$

$\begin{array}{lll}\text { No } & 137 & 24.04\end{array}$

$\begin{array}{lll}\text { Missing } & 13 & 2.28\end{array}$

Do you actively promote breastfeeding among your female house staff (residents and/or fellows), colleagues, staff, or students?

Yes

No

Missing

$481 \quad 84.39$

$78 \quad 13.68$

$11 \quad 1.93$

and description of modifiable factors associated with likelihood of longer BFD are an important step in closing the discrepancy between physician mothers' personal breastfeeding intentions and actual behavior.

In the general population, longer maternity leave, as well as adequate time and space for expressing milk once the mother returns to the workplace, have been identified as facilitators to longer BFD among working mothers. ${ }^{15-17}$ Among physician mothers, work-related factors, such as requirement to make up missed work or call that occurred as a result of pregnancy or maternity leave, maternity leave length, availability of time at work for breastfeeding/milk expression, and workplace support, are associated with BFD. ${ }^{18,19}$ However, existing studies have limitations, including limited sample size and recruiting participants from a single specialty, academic medical center, or state. ${ }^{9-14,18,19}$

The goal of this study was to assess personal breastfeeding intentions and behavior of a geographically diverse group of physician mothers from various medical specialties recruited through the Academy of Breastfeeding Medicine (ABM) and to identify characteristics associated with their BFD. This study expands on prior research to determine modifiable predictors of BFD among female physicians. The main variables of interest were BFD, defined as the age (in months) that infant was completely weaned from breast milk, and duration of $\mathrm{EBF}$, defined as the age (in months) the infant 
Table 2. Characteristics of Pregnancies AND INFANT FEEDING $(N=1,008)$

\begin{tabular}{lll}
\hline Variable & $n$ & $\%$ \\
\hline
\end{tabular}

Maternal career stage at the time of childbirth

During training (e.g., residency, fellowship) 13113.00

After completion of training

$868 \quad 86.11$

Missing

90.89

Were you required to make up any missed call your

maternity leave incurred?

Yes

$270 \quad 26.79$

No

73072.42

Missing

$\begin{array}{ll}8 & 0.79\end{array}$

Infant-feeding method at birth

Exclusive breastfeeding

Combination of breast milk and formula $\quad 95 \quad 9.34$

Not breastfeeding at all

$900 \quad 88.50$

$\begin{array}{ll}95 & 9.34 \\ 22 & 2.16\end{array}$

What was your schedule on return to work/residency?

(Postpartum Work Schedule)

Full-time

81680.95

Part-time

18117.96

Did not return

$\begin{array}{ll}7 & 0.69\end{array}$

Missing

$4 \quad 0.40$

Did you have flexibility to schedule your rotations upon return to work? (Postpartum Schedule Flexibility)

Yes $\quad 25124.91$

Somewhat

$\begin{array}{ll}404 & 40.08 \\ 161 & 15.97\end{array}$

Not available

$154 \quad 15.28$

Missing

$38 \quad 3.77$

How would you scale your mental health/emotional state during your breastfeeding period? (Depression)

Severely depressed

Mildly depressed

494.86

Not depressed at all $\quad 66966.37$

Missing

60.60

How would you rate your energy level while breastfeeding? (Energy)

Often tired

$467 \quad 46.33$

Sometimes tired

$452 \quad 44.84$

Seldom tired

$86 \quad 8.53$

Missing

30.30

How would you rate your stress level while breastfeeding? (Stress)

Very stressed

$253 \quad 25.10$

Somewhat stressed

52331.88

Seldom stressed

Missing

22622.42

$6 \quad 0.60$

Did you pump milk while working?

Yes

No

82681.94

$22 \quad 2.18$

Missing

$160 \quad 15.87$

Did you have sufficient time to express milk at work? (Time)

Never

Occasionally

$45 \quad 4.46$

Sometimes

$104 \quad 10.32$

Often

21321.13

30930.65

Always

15014.88

Missing

$187 \quad 18.55$

Did you have access to an appropriate place to express milk at work? (Space)

Never

Occasionally

$\begin{array}{ll}38 & 3.77\end{array}$

$81 \quad 8.04$

(continued)
TABle 2. (CONTINUED)

\begin{tabular}{lcc}
\hline Variable & $n$ & $\%$ \\
\hline Sometimes & 107 & 10.62 \\
Often & 208 & 20.63 \\
Always & 387 & 38.39 \\
Missing & 187 & 18.55
\end{tabular}

Were your colleagues supportive of your milk expression efforts while working? (Collegial Support)

Always opposed my efforts

Usually opposed my efforts

$\begin{array}{ll}4 & 0.40\end{array}$

Neither supportive nor oppositional

$\begin{array}{ll}16 & 1.59\end{array}$

$123 \quad 12.20$

Usually supportive

30129.86

Always supportive

$297 \quad 29.46$

Colleagues didn't know

$\begin{array}{ll}42 & 4.17\end{array}$

Not applicable

$\begin{array}{ll}9 & 0.89\end{array}$

Missing

21621.43

Was discontinuation of breastfeeding due to demands at work? Yes

Were you satisfied with the duration that you actually breastfed?

Yes

$642 \quad 63.69$

No

$128 \quad 12.70$

Somewhat

$175 \quad 17.36$

Missing

$63 \quad 6.25$

started receiving nutrition other than breast milk. Based on previous studies, we hypothesized that physician mothers would have high breastfeeding initiation rates, but lower continuation rates compared with their reported intention. We also hypothesized that certain factors, such as maternity leave duration, would be associated with longer BFD.

\section{Methods}

The Institutional Review Board at the University of Florida (UF) approved this study. We have previously described the development and prior modifications of our survey instrument. 9,10 The initial questionnaire was developed and piloted in 2008 after review of instruments used in previous breastfeeding studies among physicians. ${ }^{11-13,20}$ In 2016, we developed further survey items and converted the questionnaire to an online one that contained 33 items about maternal demographics, previous breastfeeding education, environmental factors, and breastfeeding advocacy (Table 1).

We developed three questions to explore maternal knowledge of existence of formal maternal leave policy and laws or regulations in the country of residence to encourage breastfeeding. We implemented four items to evaluate participants' self-perceived breastfeeding advocacy. In addition, we asked 46 questions pertaining to each of the participants' children to assess infant-feeding intention, infant-feeding modality at birth, maternal goal for BFD, age of infant at the time of first supplementation $(\mathrm{EBF})$ and at the time of complete weaning from breast milk (BFD), as well as workrelated factors (Table 2). The time required to complete the questionnaire depended on the participant's number of children.

Recruitment was initiated through announcements at the European ABM meeting in Lisbon, Portugal, in 2016, as well as e-mails to ABM members that contained information about the study, the online survey link, and the principal investigator contact information. Criteria for participation 
consisted of being a female physician and having at least one biological child. Eligible participants were included whether they were in training (e.g., resident or fellow) or had completed training (e.g., faculty at academic site or community practice). Participants were included regardless of their infant-feeding methods (formula, breast milk, or combination). The online questionnaire was administered through REDCap electronic data capture tools hosted at UF to generate the database for this study. ${ }^{21}$

\section{Statistical analyses}

Stata statistical software release 15 was used for data

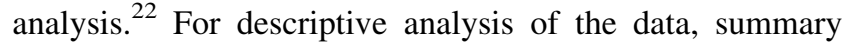
statistics (i.e., mean, standard deviation [SD], standard error of the mean [SE (mean)], median, minimum, and maximum) were used for continuous variables and proportions for categorical variables. We used the infant as the unit of analysis for calculation of breastfeeding rates because infant-feeding practices of some multiparous participants varied with different offspring. EBF and BFD were the primary dependent variables. We chose independent variables based on previous studies of breastfeeding among physician mothers, working mothers, and women in general. ${ }^{9-20}$

Independent variables included maternal covariates (e.g., age at the time of the study), environmental covariates (e.g., existence of formal maternity leave policies in maternal country of residence [maternity leave policy]), breastfeeding advocacy covariates (e.g., self-reported frequency of breastfeeding discussions with pregnant patients or new mothers [BF discussion]), and child-related covariates (e.g., BFD goal). Initial analysis involved fitting univariate regression models on each of the covariates of interest to assess association with the outcome. Inclusion of variables into the final multivariable regression model was based on variables that showed an association with the response at the univariate level.

Models for statistical inference were fitted separately for analyzing EBF and BFD, since they exhibited different distributions, requiring different statistical modeling methods. Analysis involving EBF outcome was not affected by censoring and therefore not treated using a time-to-event approach. Assumptions of normality of residuals from the EBF models were explored graphing the residuals. The BFD out- come was treated as a time-to-event analysis with censoring of observations for children still breastfeeding at the time of the survey. Thus, the observations for these children were only recorded for the length of breastfeeding at the time of the survey. Analyses of both outcomes were conducted by fitting random-intercept (mother-specific), mixed-effects regression models since mothers had several children (observations) ranging from one to six.

To test for the heterogeneity of the mother-specific effect, a likelihood ratio test was conducted. For EBF, univariate and multivariable linear-mixed models were fitted and presented using coefficients $(\beta)$, SEs, $p$-values, and $95 \%$ confidence intervals (CI) (Table 3). Univariate and multivariable methods to analyze BFD with associated potential factors were conducted by fitting proportional hazards $(\mathrm{PH})$ regression models. Results are presented using estimated coefficients and hazard ratios with SEs, $p$-values, and 95\% CI (Table 4). Missing observations were considered to be missing at random and therefore excluded from analysis.

\section{Results}

\section{Characteristics of mothers and children}

While 1,016 physicians initiated the survey, only 570 with 1,017 children met the eligibility criteria and had complete responses regarding at least one of the main outcome variables. A total of 1,008 child-specific observations had complete EBF information and 998 observations had complete BFD information. Table 1 summarizes maternal demographic characteristics. The mean participant age at the time of the study was 37.1 years $(\mathrm{SD}=5.6$; range $=26-68)$.

Participants reported a variety of countries of origin (e.g., Bangladesh, Brazil, Canada, China, Colombia, Costa Rica, Cuba, Ecuador, Germany, Guatemala, Honduras, India, Israel, Lebanon, Liberia, Mexico, Nigeria, Pakistan, Panama, Peru, Philippines, Poland, Puerto Rico, Russia, South Africa, Taiwan, Tibet, United Kingdom, United States, Venezuela, and Vietnam), but most resided in the United States at the time of the study. The majority was married and had completed their medical training. The participants had one to six children, ranging from 7 days to 38 years of age (mean of 4.4 years), at the time of the study.

Table 3. Results of the Multivariable Linear Mixed-Effect Model for Exclusive Breastfeeding Duration

\begin{tabular}{lrrrc}
\hline Independent variable & Coefficient ( $\beta)$ & $S D$ & $\mathrm{p}$ & $95 \%$ CI \\
\hline $\begin{array}{l}\text { Participant age at the time of the study } \\
\text { Not exclusively breastfeeding at birth (compared } \\
\quad \text { with exclusive breastfeeding) }\end{array}$ & -0.05 & 0.02 & 0.007 & -0.09 to -0.01 \\
$\begin{array}{l}\text { Maternal report of being "Somewhat stressed" } \\
\quad \text { compared with "Very stressed" while breastfeeding) }\end{array}$ & -2.36 & 0.20 & 0.000 & -2.74 to -1.97 \\
$\begin{array}{l}\text { Maternal report of being "Seldom stressed" (compared with } \\
\text { "Very stressed" while breastfeeding) }\end{array}$ & 0.41 & 0.18 & 0.020 & $0.06-0.75$ \\
$\begin{array}{l}\text { Breastfeeding discontinued due to work-related demands } \\
\text { (compared with not related to work-related demands) }\end{array}$ & 0.48 & 0.24 & 0.046 & $0.01-0.95$ \\
$\begin{array}{l}\text { Mother reporting satisfaction with BFD (compared with "Not satisfied") } \\
\text { Reporting occasionally having had sufficient time to express milk } \\
\text { at work (compared with "Never") }\end{array}$ & -0.40 & 0.18 & 0.025 & -0.75 to -0.05 \\
$\begin{array}{l}\text { Reported that colleagues did not know they were breastfeeding } \\
\text { (compared with "Always or usually opposing the efforts") }\end{array}$ & 0.66 & 0.24 & 0.012 & $0.13-1.07$ \\
\hline
\end{tabular}

BFD, breastfeeding duration; CI, confidence interval; SD, standard deviation. 
Table 4. Breastfeeding Duration Results From MiXed-EFFEcts Proportional Hazards REGRESSION MODELS

\begin{tabular}{|c|c|c|c|c|c|}
\hline \multirow[b]{2}{*}{ Variable } & \multicolumn{5}{|c|}{ Mixed-effects Weibull PH } \\
\hline & $H R$ & $S E$ & $\mathrm{p}$ & $95 \%$ & $C I$ \\
\hline Maternal BFD goal & 0.79 & 0.02 & 0.000 & 0.75 & 0.83 \\
\hline $\begin{array}{l}\text { Participant age at the time } \\
\text { of the study }\end{array}$ & 1.05 & 0.02 & 0.021 & 1.01 & 1.10 \\
\hline Maternity leave length & 0.95 & 0.02 & 0.003 & 0.91 & 0.98 \\
\hline \multicolumn{6}{|c|}{ BF work laws (compared with "No") } \\
\hline Yes & 0.56 & 0.15 & 0.035 & 0.32 & 0.96 \\
\hline Don't know & 1.10 & 0.38 & 0.773 & 0.56 & 2.16 \\
\hline \multicolumn{6}{|c|}{ Child order (compared with "First child") } \\
\hline Second child & 0.69 & 0.09 & 0.007 & 0.53 & 0.9 \\
\hline Third + child & 0.52 & 0.13 & 0.009 & 0.32 & 0.8 \\
\hline \multicolumn{6}{|c|}{ Depression (compared with "Severely depressed") } \\
\hline Mildly depressed & 0.55 & 0.20 & 0.096 & 0.27 & 1.11 \\
\hline Not depressed at all & 0.38 & 0.15 & 0.013 & 0.18 & 0.82 \\
\hline $\begin{array}{l}\text { Breastfeeding } \\
\text { discontinued due to } \\
\text { work-related demands } \\
\text { (compared with } \\
\text { "Breastfeeding not } \\
\text { discontinued due to } \\
\text { work-related demands" }\end{array}$ & 1.80 & 0.40 & 0.007 & 1.17 & 2.78 \\
\hline \multicolumn{6}{|c|}{ Maternal satisfaction with BFD (compared with "No") } \\
\hline Yes & 0.02 & 0.01 & 0.000 & 0.01 & \\
\hline Somewhat & 0.16 & 0.05 & 0.000 & 0.09 & 0.3 \\
\hline
\end{tabular}

$\mathrm{BFD}$, breastfeeding duration; BF work laws, laws or regulations in the country to encourage breastfeeding among working mothers; CI, confidence interval; Depression, maternal mental health/emotional state during breastfeeding period; HR, hazards ratio; SE, standard error.

\section{Breastfeeding intentions and behavior}

Most of the children $(n=868 ; 86.1 \%)$ were born after the participants had completed their medical training (Table 2). The mean length of maternity leave was 9.97 weeks (SD 6.67, range 0-64) and of paid maternity leave was 5.80 weeks
(SD 5.64, range 0-54). Of the 972 responses provided for maternal goal for BFD, 759 (78.09\%) stated a goal of 12 months or more. Immediately after birth, $88.50 \%(900 / 1,017)$ of infants were breastfeeding exclusively and 97.84\% (995 out of 1,017 ) received at least some breast milk. Using the infant as the unit of analysis, the breastfeeding initiation rate was $97.84 \%$, and continuation rates were $85.45 \%$ at 6 months and $55.36 \%$ at 12 months (Fig. 1). EBF rates were $88.50 \%$ at birth, $76.30 \%$ at 3 months, and $40.90 \%$ at 6 months. At the time of the study, 235 children $(23.55 \%)$ were still receiving breast milk.

The participants reported achieving their BFD goals in 583 $(58.42 \%)$ instances. When we excluded children breastfeeding at the time of the study, participants met their BFD duration goals in $61.60 \%(470 / 763)$ of cases. The average BFD was higher for participants who achieved their BFD goal, compared with those who did not meet their BFD goal $(\beta=6.56+0.46, C I=5.66-7.46)$.

\section{Breastfeeding advocacy}

Of the 557 participants who responded to the question, 420 (75.4\%) reported that they felt they actively promote breastfeeding among their women patients (Table 1). Of the 559 respondents to the question, $481(86.0 \%)$ reported that they actively promote breastfeeding among female house staff (residents and/or fellows), colleagues, staff, or students. Of the 554 participants who responded to the question, $338(61.0 \%)$ reported that they always or usually talk about breastfeeding with a typical pregnant patient or new mother, $114(20.6 \%)$ reported "sometimes" doing so, and 102 (18.4\%) reported never. Of the 551 respondents, $208(37.7 \%)$ reported strongly encouraging pregnant patients or new mothers to breastfeed, $227(41.2 \%)$ reported encouraging breastfeeding, $116(21.1 \%)$ reported neither encouraging nor discouraging, and 7 (1.3\%) reported discouraging or strongly discouraging.

\section{Inferential analysis}

Exclusive breastfeeding. Linear mixed-model univariate analysis indicated a statistically significant negative

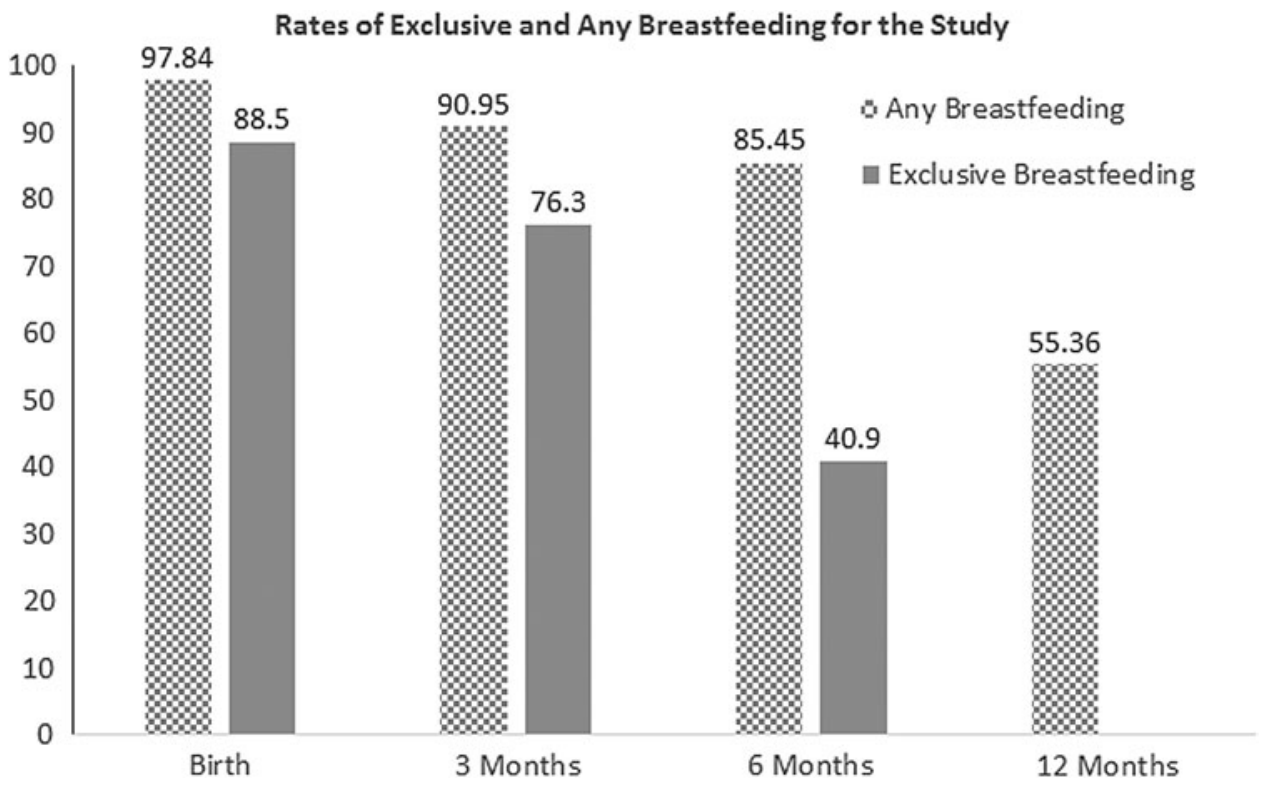

FIG. 1. Rates of exclusive and any breastfeeding for the study. Using the infant as the unit of analysis, we calculated breastfeeding initiation rates at birth, continuation rates at 6 and 12 months, and exclusive breastfeeding rates at birth, 3, and 6 months. 
association between EBF and participant age, not exclusively breastfeeding at birth, breastfeeding discontinuation due to work-related demands, frequency of handing over pager while expressing milk (pager), maternal mental health/emotional state while breastfeeding (depression), and maternal level of stress while breastfeeding (stress). On the contrary, there was a statistically significant positive association between EBF and maternal BFD goal, number of children, child order, maternal career stage, existence of laws to encourage breastfeeding (BF laws), existence of laws to encourage breastfeeding among working mothers (BF work laws), BF discussion, self-reported breastfeeding advocacy with pregnant patients or new mothers (BF encouragement), selfreported breastfeeding promotion among women patients (BF promotion), and self-reported breastfeeding promotion among house staff, colleagues, staff, or students.

The following covariates also had a statistically significant positive association with EBF: maternal level of energy while breastfeeding (energy), maternal satisfaction with BFD, availability of time to express milk/breastfeed at work (time), access to appropriate place to express milk/breastfeed at work (space), and perceived level of support for breastfeeding efforts from colleagues (collegial support) and attending physicians (attending support). After adjusting for other covariates in a multivariable linear mixed-effect model, only seven variables continued to have a statistically significant association with EBF: younger participant age at the time of the study, infant-feeding method at birth, lower level of stress, breastfeeding discontinuation not due to work-related demands, heightened maternal satisfaction with BFD, time, and collegial support (Table 3).

The average EBF decreased with participant age. EBF at birth was associated with longer EBF. Compared with being "very stressed" while breastfeeding, responses of "somewhat stressed" and "seldom stressed" were both associated with longer EBF. Reporting that breastfeeding discontinuation was due to work-related demands was associated with shorter EBF. Longer EBF was associated with increasing levels of maternal satisfaction with BFD. Availability of time at work to express breast milk/breastfeed and perceived level of support from colleagues for breastfeeding efforts had a statistically significant positive association with EBF. Even mothers who reported their colleagues did not know they were breastfeeding had longer EBF compared with the mothers with colleagues who always or usually opposed their breastfeeding efforts.

The intra-mother correlation was high, estimated to be $0.72 \pm 0.03,95 \%$ CI $0.66-0.78$ and the between-mother variance of EBF was 2.4 months $\pm 0.24,95 \%$ CI $1.98-2.92$. The likelihood ratio test for mother-specific variance indicates that this variance could not have been ignored (not 0 ) with a chi-square value $\chi_{1}^{2}=187.15$ and $p$-value $<0.001$.

\section{Breastfeeding duration}

The univariate mixed-effects Weibull regression model indicated a statistically significant association between BFD and the following covariates: participant age, maternal BFD goal, maternity leave length, paid maternity leave duration, child order (first child versus second child and third child +), maternity leave policy, BF laws, BF work laws, formal breastfeeding education during residency, breastfeeding train- ing outside formal education, including counseling about breastfeeding a participant's own child (informal training), $\mathrm{BF}$ discussion, $\mathrm{BF}$ promotion, $\mathrm{BF}$ encouragement, selfreported breastfeeding promotion among house staff, colleagues, staff, or students, postpartum work schedule (e.g., full-time, part-time), postpartum schedule flexibility, infantfeeding method at birth, depression, energy, stress, breastfeeding discontinuation due to work-related demands, maternal satisfaction with BFD, time, space, collegial support, and return to work on or after 2003.

After fitting multivariable $\mathrm{PH}$ regression model and adjusting for all other covariates in the model, only eight variables continued to have a statistically significant association with BFD: younger participant age at the time of the study, longer maternal BFD goal, longer maternity leave duration, BF work laws, later child order, lower level of maternal depression, breastfeeding discontinuation not due to work-related demands, and heightened maternal satisfaction with BFD (Table 4).

Similar to EBF, participant age was negatively associated with BFD, with a $1 \%$ decrease in BFD for each year increase in participant's age at the time of the study. Maternal goal for BFD had a high correlation with BFD, with an average $4 \%$ increase in BFD for each month increase in maternal goal. BFD increased by $1 \%$ for every extra week of maternity leave. The median BFD was 12 months for mothers who responded "No" to "Does your country have any laws or regulations to encourage breastfeeding among working mothers?" as well as mothers who responded "Don't know" $(p=0.773)$, compared with median BFD of 14 months for mothers who responded "Yes" $(p=0.035)$.

BFD was on average shorter for first-born children. Compared with first-born children, second-born children were breastfed about $6 \%$ longer and the third or higher born children about $11 \%$ longer. Reporting not being depressed at all while breastfeeding was associated with $16 \%$ increase in BFD, compared with severe depression. Breastfeeding discontinuation due to work-related demands was associated with $80 \%$ increased hazard of having a short BFD according to the PH model. Compared with the response of "No" to the question, "Were you satisfied with the duration that you actually breastfed?" Both "Yes" and "Somewhat" responses were associated with longer BFD.

\section{BFD goal achievement}

The mean age of participants who reported meeting their BFD goal (38.2 \pm 6.6$)$ was slightly higher than those who reported not achieving their goal $(37.6+5.5)$. However, the difference between the two groups was not statistically significant $(p=0.097)$. Similarly, there were not statistically significant differences in duration of maternity leave or paid maternity leave and number of children between the two groups.

Linear mixed-model univariate analysis indicated a statistically significant association between achievement of BFD goal and the following covariates: formal breastfeeding education during residency, $\mathrm{BF}$ laws, BF work laws, BF encouragement, BF promotion, and maternal career stage at the time of childbirth. After adjusting for other covariates in a multivariable linear mixed-effect model, the association with the following variables remained statistically significant: $\mathrm{BF}$ laws, BF encouragement, and maternal career stage at the time of childbirth (Table 5). 
Table 5. Results of the Multivariable Linear Mixed-Effect Model for Achieving BreastFEEDING Duration Goals

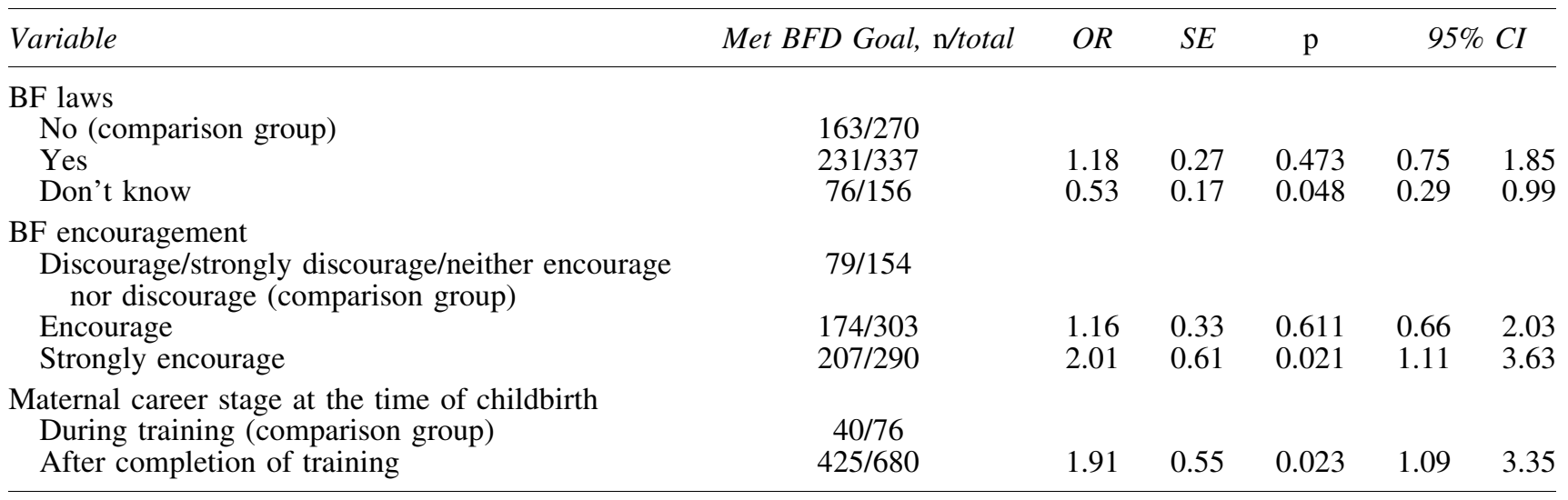

BFD, breastfeeding duration; BF encouragement, self-reported breastfeeding advocacy with pregnant patients or new mothers; BF laws, existence of laws in the maternal country of residence to encourage breastfeeding; CI, confidence interval; OR, odds ratio; SE, standard error.

\section{Discussion}

Consistent with other physician studies, ${ }^{9-14,23-26}$ we found high breastfeeding initiation rates in this study. We note that the breastfeeding rates in this study surpass those of the general population and meet the Healthy People 2020 breastfeeding objectives. ${ }^{27,28}$ However, our data also demonstrate that while $98 \%$ of infants were breastfed at birth and intent to breastfeed for at least 12 months was $78 \%$, only $55 \%$ of infants continued to receive breast milk at 12 months of age. This discrepancy and the work-related factors associated with EBF and BFD suggest that work-related factors not only influence physician mothers' breastfeeding behavior but also might have a larger impact than their education and intentions on their BFD.

Potentially modifiable factors with statistically significant association with BFD or EBF include infant-feeding method at birth, maternity leave duration, laws to support breastfeeding among working mothers, maternal levels of stress and depression while breastfeeding, availability of time to express milk/breastfeed at work, and collegial support. We found statistically significant associations between BFD of physician mothers and duration of maternity leave as well as maternal BFD goal, consistent with previous studies. ${ }^{18,19}$ However, we did not find an association with maternal requirement to make up missed work or call that occurred as a result of pregnancy or maternity leave. ${ }^{18,19}$ Availability of time at work to express milk/breastfeed and collegial support of breastfeeding efforts had a statistically significant association with EBF, but not BFD.

Not surprisingly, maternal satisfaction with BFD correlated with both EBF and BFD. We believe that mothers who were able to achieve their BFD goal or come closer to it were more likely to be satisfied with their BFD. Reporting that discontinuation of breastfeeding was due to work-related demands was associated with shorter duration of both exclusive and any breastfeeding. The negative association between participants' age at the time of study and both EBF and BFD might reflect generational changes in infant-feeding beliefs and behavior as well as working environments of physicians. Infant-feeding practices in 1978 may not be equivalent to those in 2016. The association between BFD and child order might reflect experiential learning of mothers with each child.

Having a child after completion of medical training was associated with higher likelihood of achieving personal BFD goals. This correlation might reflect the extent of maternal control of her schedule as well as work environment. Physicians who reported strongly encouraging their pregnant or recently pregnant patients to breastfeed were more likely to report meeting their personal BFD goal, compared with physicians who reported discouraging, strongly discouraging, and neither encouraging or discouraging. While we did not find a statistically significant difference in the proportion of mothers who achieved their personal BFD goals between the groups that reported existence of laws in their country of residence and those who responded "No" to the question, a response of "Don't Know" was associated with lower likelihood of meeting personal BFD goals.

Strengths of our study include its large sample size and recruitment of women physicians from a variety of disciplines. However, our final study cohort did not have the international diversity that we had hoped for when designing this study. Also, our cross-sectional study cannot determine causality. For example, reported knowledge about workplace policies does not necessarily predate the participants' infantfeeding behaviors. As reported by Dixit et al., many physicians, particularly physicians in training, are unaware of policies related to maternity leave or breastfeeding support. ${ }^{29}$

Other potential limitations of our study include recall bias since we collected data on infant-feeding practices reported over a span of nearly 40 years. Both the recall bias and change in infant-feeding practices might have attributed to the inverse correlation between participant age and outcomes of $\mathrm{EBF}$ and BFD. Our findings are based on self-reported data and it is possible that respondents may have given answers they perceived to be more acceptable. This social desirability bias may have resulted in overestimations or underestimations of perception-based questions.

Our data may also have been skewed by response and selfselection bias as our participants were volunteers contacted through the ABM, representing physicians with knowledge and favorable perception of breastfeeding, who might not be 
representative of all physicians, thereby limiting the generalizability of our results to non-ABM members. For example, the commitment of our respondents to breastfeeding might have lessened the impact of some variables, such as work environment, which might have a stronger correlation with breastfeeding behavior among non-ABM members. Also, since intent has a great impact on breastfeeding behavior, the results from the highly motivated and committed physicians involved in this study might not be generalizable to all physicians. Furthermore, we do not have information available regarding the proportion of respondents compared with physicians who would have been eligible to participate in our study and received the e-mail (e.g., women, parents). Therefore, the proportions reported in this study might not even be representative of the ABM members.

While we believe our participants reported their infant receiving any breast milk as breastfeeding, the breastfeeding rates as well as duration of breastfeeding and EBF could underrepresent the actual breastfeeding behavior of our participants since we did not use the term "exclusive pumping" in our questionnaire. Furthermore, the reliability and accuracy of our questions for assessing maternal depression, stress, and fatigue while breastfeeding have not been assessed previously. Finally, we did not collect data regarding participants' race/ethnicity.

Despite these limitations, our study is the first reported international survey study of infant-feeding intentions and behavior among physicians (MEDLINE; 1946-December 2018; English language; search terms of "physicians," and "breastfeeding") and may help provide information to shape the policy in relation to physicians. Examples of workplace policies to support physician mothers in achieving their breastfeeding goals would be longer maternity leave and workplace support of breastfeeding efforts, including providing adequate breaks in the schedule for physicians to breastfeed and/or express milk. Since there are global differences in maternity leave length and workplace policy, future studies to compare infant-feeding behavior of physician mothers in different countries would be informative.

\section{Conclusion}

We found high breastfeeding rates in our study cohort contacted through the ABM that represents physicians with knowledge and favorable perception of breastfeeding. Maternal intentions correlated with physician mothers' breastfeeding practices, but did not seem sufficient for achieving their personal BFD objectives. Rather, breastfeeding maintenance seems to be determined by interaction of work-related factors, such as maternity leave duration, with personal ones, such as intent.

Longer maternity leave, laws and regulations to encourage breastfeeding among working mothers, and enhanced workplace support for the breastfeeding physician and her breastfeeding efforts might improve physician mothers' breastfeeding maintenance after return to work. Modifiable work-related factors that might enhance physician mothers' EBF behavior include dedicated time to express milk/breastfeed at work and increased workplace support. In addition, while multifactorial in nature, the identification of indicators of stress and depression may prompt change and addition of resources to help offload stressors for physician mothers attempting to maintain breastfeeding after return to work.

\section{Acknowledgment}

We thank Dr. Denis Valle for his help with the statistical methods.

\section{Disclosure Statement}

No competing financial interests exist.

\section{Funding Information}

This work was partially supported by the NIH grant no. 1UL1TR000064 from the National Center for Advancing Translational Sciences and by the Clinical and Translational Science Institute, NIH grant no. 1UL1RR029890.

\section{References}

1. Section on Breastfeeding. Breastfeeding and the use of human milk Pediatrics 2012;129:e827-e841.

2. Victora CG, Bahl R, Barros AJ, et al. Breastfeeding in the 21st century: Epidemiology, mechanisms, and lifelong effect. Lancet 2016;387:475-490.

3. Rollins NC, Bhandari N, Hajeebhoy N, et al. Lancet Breastfeeding Series: Why invest, and what it will take to improve breastfeeding practices in less than a generation. Lancet 2016;387:491-504.

4. American Academy of Pediatrics. Infant Food and Feeding. 2019. Available at www.aap.org/en-us/advocacy-andpolicy/aap-health-initiatives/HALF-Implementation-Guide/ Age-Specific-Content/Pages/Infant-Food-and-Feeding.aspx (accessed September 20, 2019).

5. Lawrence RA. Practices and attitudes toward breastfeeding among medical professionals. Pediatrics 1982;70: 912-920.

6. Brodribb W, Fallon A, Jackson C, et al. The relationship between personal breastfeeding experience and the breastfeeding attitudes, knowledge, confidence and effectiveness of Australian GP registrars. Matern Child Nutr 2008;4:264274.

7. Power ML, Locke E, Chapin J, et al. The effort to increase breast-feeding. Do obstetricians, in the forefront, need help? J Reprod Med 2003;48:72-78.

8. Pascoe JM, Pletta K, Beasley J, et al. Best start breastfeeding promotion campaign. Pediatrics 2002;109:170.

9. Sattari M, Levine D, Bertram A, et al. Breastfeeding intentions of female physicians. Breastfeed Med 2010;5:297302.

10. Sattari M, Levine D, Neal D, et al. Personal breastfeeding behavior of physician mothers is associated with their clinical breastfeeding advocacy. Breastfeed Med 2013;8: 31-37.

11. Miller N, Miller D, Chism M. Breastfeeding practices among resident physicians. Pediatrics 1996;98:434-437.

12. Arthur CR, Saenz RB, Replogle WH. The employmentrelated breastfeeding decisions of physician mothers. J Miss State Med Assoc 2003;44:383-387.

13. Arthur CR, Saenz RB, Replogle WH. Personal breastfeeding behaviors of female physicians in Mississippi. South Med J 2003;96:130-135.

14. Kacmar JE, Taylor JS, Nothnagle M, et al. Breastfeeding practices of resident physicians in Rhode Island. Med Health R I 2006;89:230-231.

15. Guendelman S, Kosa JL, Pearl M, et al. Juggling Work and breastfeeding: Effects of maternity Leave and occupational Characteristics. Pediatrics 2009;123:e38-e46. 
16. Jacknowitz A. The role of workplace characteristics in breastfeeding practices. Women Health 2008;47:87-111.

17. Johnston ML, Esposito N. Barriers and facilitators for breastfeeding among working women in the United States. J Obstet Gynecol Neonatal Nurs 2007;36:9-20.

18. Sattari M, Serwint JR, Neal D, et al. Work-place predictors of duration of breastfeeding among female physicians. J Pediatr 2013;163:1612-1617.

19. Sattari M, Serwint JR, Shuster JJ, et al. Infant-feeding intentions and practices of internal medicine physicians. Breastfeed Med 2016;11:173-179.

20. Freed GL, Clark SJ, Sorenson J, et al. National assessment of physicians' breastfeeding knowledge, attitudes, training, and experience. JAMA 1995;273:472-476.

21. Harris PA, Taylor R, Thielke R, et al. Research electronic data capture (REDCap) — A metadata-driven methodology and workflow process for providing translational research informatics support. J Biomed Inform 2009;42:377-381.

22. StataCorp. Stata Statistical Software: Release 15. College Station, TX: StataCorp LLC, 2017.

23. Orth TA, Drachman D, Habak P. Breastfeeding in obstetrics residency: Exploring maternal and colleague resident perspectives. Breastfeed Med 2013;8:394-400.

24. Riggins C, Rosenman MB, Szucs KA. Breastfeeding experiences among physicians. Breastfeed Med 2012;7:151154.

25. Hamilton AR, Tyson MD, Braga JA, et al. Childbearing and pregnancy characteristics of female orthopaedic surgeons. J Bone Joint Surg Am 2012;6;94:e77.
26. Lerner LB, Baltrushes RJ, Stolzmann KL, et al. Satisfaction of women urologists with maternity leave and childbirth timing. J Urol 2010;183:282-286.

27. Centers for Disease Control and Prevention. Breastfeeding Report Card. 2018. Available at www.cdc.gov/breast feeding/data/reportcard.htm (accessed January 20, 2019).

28. US Department of Health and Human Services. Healthy People 2020 Objectives: Maternal, Infant, and Child Health: MICH-21. 2014. Available at www.healthypeople .gov/2020/topics-objectives/topic/maternal-infant-and-childhealth/objectives (accessed January 20, 2019).

29. Dixit A, Feldman-Winter L, Szucs KA. Parental leave policies and pediatric trainees in the United States. J Hum Lact 2015;31:240-248.

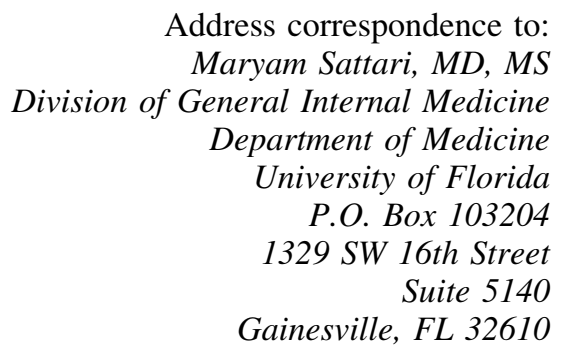

USA

E-mail: maryam.sattari@medicine.ufl.edu 